\title{
Quantum scalar corrections to the gravitational potentials on de Sitter background
}

\section{Sohyun Park, ${ }^{a}$ Tomislav Prokopec $^{b}$ and R.P. Woodard ${ }^{c}$}

${ }^{a}$ Korea Astronomy and Space Science Institute, Daejeon, 305-348, Republic of Korea

${ }^{b}$ Institute of Theoretical Physics, Spinoza Institute and the Center for Extreme Matter and Emergent Phenomena (EMME $\Phi$ ), Utrecht University, Leuvenlaan 4, 3584 CE Utrecht, The Netherlands

${ }^{c}$ Department of Physics, University of Florida, Gainesville, FL 32611, U.S.A.

E-mail: spark1@kasi.re.kr, T.Prokopec@uu.nl, woodard@phys.ufl.edu

ABSTRACT: We employ the graviton self-energy induced by a massless, minimally coupled (MMC) scalar on de Sitter background to compute the quantum corrections to the gravitational potentials of a static point particle with a mass $M$. The Schwinger-Keldysh formalism is used to derive real and causal effective field equations. When evaluated at the one-loop order, the gravitational potentials exhibit a secular decrease in the observed gravitational coupling $G$. This can also be interpreted as a (time dependent) anti-screening of the mass $M$.

KEYWORDS: Models of Quantum Gravity, Effective field theories

ARXIV EPRINT: 1510.03352 


\section{Contents}

1 Introduction 1

2 Schwinger-Keldysh effective field equations 2

2.1 Preliminaries 2

2.2 Effective field equations 3

2.3 Perturbative solution 4

2.4 Schwinger-Keldysh formalism 4

3 Quantum corrected gravitational potentials $\quad 6$

3.1 Classical solutions 6

$\begin{array}{lll}3.2 & \text { Computing the one-loop source integrals } & 7\end{array}$

$\begin{array}{lll}4 & \text { Discussion } & 10\end{array}$

A Evaluating the source integral $\quad 12$

$\begin{array}{lll}\text { A.1 Late time limit of the gravitational potentials } & 14\end{array}$

\section{Introduction}

Studies of quantum loop corrections to the gravitational potentials in flat space background have a long history [1-16]. These studies are typically based on computing the scattering amplitude for two massive particles and then solving the inverse scattering problem to reconstruct a Newtonian potential which would produce the same scattering amplitude in quantum mechanics. This technique is well-tested and has the tremendous advantage of being independent of the choice of gauge and of field variable. However, it seems artificially restricted to asymptotic scattering problems, as compared with the time-dependent effects which can be explored using the classical field equations. And its application to cosmology seems inappropriate because the formal S-matrix which can be defined for massive scalars on de Sitter [17] is not observable.

A more suitable technique for time-dependent sources and cosmological backgrounds is the Schwinger-Keldysh, or in-in, formalism [18-22], which provides expectation values of operators instead of in-out matrix elements. The authors have previously solved the Schwinger-Keldysh effective field equations to work out quantum corrections (from a massless, minimally coupled (MMC) scalar) to the two potentials of a point mass on flat space background [23-25]. When graviton and gauge particles start to appear in loops the problem of gauge dependence must be faced, but that is not an issue here. And it should be noted that the Schwinger-Keldysh results are consistent with those derived using conventional scattering techniques. They also furnish an essential correspondence limit for the current computation in de Sitter. 
De Sitter space is of particular interest in cosmology as a paradigm for the background of primordial inflation. A generic prediction of inflation is that the quantum fluctuations of MMC scalars and gravitons are amplified and preserved to late times so that they seed large scale structure formation [26-33]. This is a tree order effect, but the same quantum fluctuations inevitably give rise to loop effects which have been studied in recent years [34-66]. The purpose of this paper is to learn how a loop of MMC scalars changes the gravitational potentials of a point mass on de Sitter. This involves three tasks:

1. Compute and renormalize the one-loop contribution to the graviton self-energy $-i\left[\mu \nu \Sigma^{\rho \sigma}\right]\left(x ; x^{\prime}\right)$ from a MMC scalar on de Sitter background;

2. Convert the in-out self-energy to the retarded one of the Schwinger-Keldysh formalism,

$$
\left[{ }^{\mu \nu} \Sigma^{\rho \sigma}\right]\left(x ; x^{\prime}\right) \rightarrow\left[{ }^{\mu \nu} \Sigma_{\text {Ret }}^{\rho \sigma}\right]\left(x ; x^{\prime}\right) ;
$$

3. Solve the quantum corrected, linearized Einstein field equation

$$
\mathcal{D}^{\mu \nu \rho \sigma} \kappa h_{\rho \sigma}(x)-\int d^{4} x^{\prime}\left[\mu \nu \Sigma_{\mathrm{Ret}}^{\rho \sigma}\right]\left(x ; x^{\prime}\right) \kappa h_{\rho \sigma}\left(x^{\prime}\right)=8 \pi G \mathcal{T}_{\text {lin }}^{\mu \nu}(x) .
$$

Here $\mathcal{D}^{\mu \nu \rho \sigma} \kappa h_{\rho \sigma}(x)$ is derived by expanding the gravitational side of the Einstein equation, $\left(R^{\mu \nu}+\Lambda g^{\mu \nu}-\frac{1}{2} g^{\mu \nu} R\right) \sqrt{-g}$, about de Sitter background to first order in the metric perturbation, $\delta g_{\rho \sigma}(x) \equiv a^{2}(t) \kappa h_{\rho \sigma}(x)$, where $a=a(t)$ is the scale factor, $\kappa^{2}=16 \pi G$ is the quantum gravitational loop counting parameter, $G$ is the Newton constant, and $\mathcal{T}_{\text {lin }}^{\mu \nu}(x)$ is the linearized stress-energy tensor density.

The first two steps have been already performed in the ref. [55] and we summarize the results in section II. Section III is devoted to the last step, that is to solving the SchwingerKeldysh effective field equations for the graviton field sourced by a static point mass. Our discussion comprises section IV, and some tedious technical details from section III have been subsumed to an appendix.

\section{Schwinger-Keldysh effective field equations}

The point of this section is to present the Schwinger-Keldysh effective field equations which we will solve in the next section. We first set up the background geometry and define the graviton field as a perturbation around this background. We then give the inout effective field equations derived in [55] and discuss how to solve them perturbatively. Finally, we explain why it is more appropriate to convert to in-in equations for cosmological backgrounds such as de Sitter, and we make the conversion.

\section{$2.1 \quad$ Preliminaries}

We consider the Lagrangian of gravity plus a MMC scalar,

$$
\mathcal{L}=\frac{1}{16 \pi G}(R-2 \Lambda) \sqrt{-g}-\frac{1}{2} \partial_{\mu} \phi \partial_{\nu} \phi g^{\mu \nu} \sqrt{-g},
$$


where $G$ is Newton's constant, $R$ is the Ricci scalar and $\Lambda$ is the cosmological constant. Our computation is based on perturbation theory in the Poincaré patch of de Sitter space

$$
d s^{2}=\hat{g}_{\mu \nu} d x^{\mu} d x^{\nu}=a^{2}(\eta) \eta_{\mu \nu} d x^{\mu} d x^{\nu} .
$$

The coordinate ranges are

$$
-\infty<x^{0} \equiv \eta<0 \quad, \quad-\infty<x^{i}<+\infty .
$$

Here the scale factor depends on conformal time $\eta$ as, $a=-1 / H \eta$ and the Hubble parameter $H=\sqrt{\frac{1}{3} \Lambda}$ is constant. It is also useful to employ the de Sitter length function,

$$
y\left(x ; x^{\prime}\right) \equiv H^{2} a a^{\prime}\left[\left\|\vec{x}-\vec{x}^{\prime}\right\|^{2}-\left(\left|\eta-\eta^{\prime}\right|-i \varepsilon\right)^{2}\right],
$$

where $a \equiv a(\eta)$ and $a^{\prime} \equiv a\left(\eta^{\prime}\right)$. Note that $y\left(x ; x^{\prime}\right)$ is related to geodesic distance on de Sitter $\ell\left(x ; x^{\prime}\right)$ as, $\left.y\left(x ; x^{\prime}\right)\right|_{\epsilon=0}=4 \sin ^{2}\left(H \ell\left(x ; x^{\prime}\right) / 2\right)$. We define the graviton field $h_{\mu \nu}$ by subtracting the background from the full metric and then conformally rescaling,

$$
h_{\mu \nu}(x) \equiv \frac{g_{\mu \nu}(x)-\hat{g}_{\mu \nu}(x)}{\kappa a^{2}} \quad \text { or } \quad g_{\mu \nu}(x)=a^{2}\left[\eta_{\mu \nu}+\kappa h_{\mu \nu}(x)\right] \equiv a^{2} \widetilde{g}_{\mu \nu}(x),
$$

where $\kappa^{2} \equiv 16 \pi G$ is the loop-counting parameter of quantum gravity.

\subsection{Effective field equations}

Varying the one-particle irreducible (1PI) effective action corresponding to the Lagrangian (2.1) with respect to the graviton field $h_{\mu \nu}$, and retaining only the linear terms gives,

$$
\mathcal{D}^{\mu \nu \rho \sigma} h_{\rho \sigma}(x)-\int d^{4} x^{\prime}\left[{ }^{\mu \nu} \Sigma^{\rho \sigma}\right]\left(x ; x^{\prime}\right) h_{\rho \sigma}\left(x^{\prime}\right)=\frac{\kappa}{2} \mathcal{T}_{\operatorname{lin}}^{\mu \nu}(x) .
$$

Here the Lichnerowicz operator for de Sitter is [55, 67],

$$
\begin{aligned}
\mathcal{D}^{\mu \nu \rho \sigma}= & \frac{1}{2} a^{2}\left[\left(\eta^{\mu(\rho} \eta^{\sigma) \nu}-\eta^{\mu \nu} \eta^{\rho \sigma}\right) \partial^{2}+\eta^{\mu \nu} \partial^{\rho} \partial^{\sigma}+\eta^{\rho \sigma} \partial^{\mu} \partial^{\nu}-2 \partial^{(\mu} \eta^{\nu)(\rho} \partial^{\sigma)}\right] \\
& +H a^{3}\left[\left(\eta^{\mu \nu} \eta^{\rho \sigma}-\eta^{\mu(\rho} \eta^{\sigma) \nu}\right) \partial_{0}-2 \eta^{\mu \nu} \delta_{0}^{(\rho} \partial^{\sigma)}+2 \delta_{0}^{(\rho} \eta^{\sigma)(\mu} \partial^{\nu)}\right]+3 H^{2} a^{4} \eta^{\mu \nu} \delta_{0}^{\rho} \delta_{0}^{\sigma}
\end{aligned}
$$

Quantum corrections come from the graviton self-energy whose general form is,

$$
\begin{aligned}
-i\left[{ }^{\mu \nu} \Sigma^{\rho \sigma}\right]\left(x ; x^{\prime}\right)= & \mathcal{F}^{\mu \nu}(x) \times \mathcal{F}^{\rho \sigma}\left(x^{\prime}\right)\left[F_{0}\left(x ; x^{\prime}\right)\right]+\mathcal{G}^{\mu \nu}(x) \times \mathcal{G}^{\rho \sigma}\left(x^{\prime}\right)\left[G_{0}\left(x ; x^{\prime}\right)\right] \\
& +\mathcal{F}^{\mu \nu \rho \sigma}\left[F_{2}\left(x ; x^{\prime}\right)\right]+\mathcal{G}^{\mu \nu \rho \sigma}\left[G_{2}\left(x ; x^{\prime}\right)\right] .
\end{aligned}
$$

The four projection operators $\mathcal{F}^{\mu \nu}, \mathcal{G}^{\mu \nu}, \mathcal{F}^{\mu \nu \rho \sigma}$ and $\mathcal{G}^{\mu \nu \rho \sigma}$ and one loop results for the corresponding structure functions $F_{0}, G_{0}, F_{2}$, and $G_{2}$ are given in ref. [55].

It is convenient to re-express the action of the Lichnerowicz operator on the graviton by extracting the scale factor $a,{ }^{1}$

$$
E^{\mu \nu} \equiv \mathcal{D}^{\mu \nu \rho \sigma} h_{\rho \sigma}(x)=\partial_{\alpha}\left[a^{2} \mathcal{L}^{\mu \nu \rho \sigma \alpha \beta} \partial_{\beta} h_{\rho \sigma}(x)\right]+\partial_{\alpha}\left[H a^{3} \eta^{\mu \nu} h^{\alpha 0}\right]-H a^{3} \eta^{0(\mu} \partial^{\nu)} h,
$$

\footnotetext{
${ }^{1}$ Due to an error, only the first term in (2.9) is found in ref. [55].
} 
where the Lichnerowicz tensor factor $\mathcal{L}^{\mu \nu \rho \sigma \alpha \beta}$ is

$$
\mathcal{L}^{\mu \nu \rho \sigma \alpha \beta} \equiv \frac{1}{2} \eta^{\alpha \beta}\left[\eta^{\mu(\rho} \eta^{\sigma) \nu}-\eta^{\mu \nu} \eta^{\rho \sigma}\right]+\frac{1}{2} \eta^{\mu \nu} \eta^{\rho(\alpha} \eta^{\beta) \sigma}+\frac{1}{2} \eta^{\rho \sigma} \eta^{\mu(\alpha} \eta^{\beta) \nu}-\eta^{\alpha(\rho} \eta^{\sigma)(\mu} \eta^{\nu) \beta} .
$$

For the quantum correction, we extract the unprimed derivatives from the $x^{\prime \mu}$ integration and partially integrate the primed derivatives, bringing the effective field equation (2.6) to the form

$$
\begin{aligned}
E^{\mu \nu}= & \frac{\kappa}{2} \mathcal{T}_{\operatorname{lin}}^{\mu \nu}(x)+\mathcal{F}^{\mu \nu} \int d^{4} x^{\prime} i F_{0}\left(x ; x^{\prime}\right) \widetilde{R}_{\operatorname{lin}}\left(x^{\prime}\right)+\mathcal{G}^{\mu \nu} \int d^{4} x^{\prime} i G_{0}\left(x ; x^{\prime}\right) \widetilde{\widetilde{R}}_{\operatorname{lin}}\left(x^{\prime}\right) \\
& -2 \partial_{\alpha} \partial_{\beta} \int d^{4} x^{\prime}\left[i F_{2}\left(x ; x^{\prime}\right) \widetilde{C}_{\operatorname{lin}}^{\mu \alpha \beta}\left(x^{\prime}\right)+i G_{2}\left(x ; x^{\prime}\right) \widetilde{\widetilde{C}}_{\operatorname{lin}}^{\mu \alpha \nu \beta}\left(x^{\prime}\right)\right] \\
& +\left[\eta^{\mu \nu} \partial_{k} \partial_{\ell}-2 \delta_{(k}^{(\mu} \partial^{\nu)} \partial_{\ell)}+\delta_{k}^{(\mu} \delta_{\ell}^{\nu)} \partial^{2}\right] \int d^{4} x^{\prime} i G_{2}\left(x ; x^{\prime}\right) \widetilde{C}_{\operatorname{lin}}^{0 k 0 \ell}\left(x^{\prime}\right)
\end{aligned}
$$

Here $\widetilde{R}_{\text {lin }}$ and $\widetilde{C}_{\text {lin }}^{\alpha \beta \gamma \delta}$ are the linearized Ricci scalar and Weyl tensor of the conformally rescaled metric. $\widetilde{\widetilde{R}}_{\text {lin }}$ and $\overline{\widetilde{C}}_{\text {lin }}^{\alpha \beta \gamma \delta}$ are their purely spatial parts, respectively.

\subsection{Perturbative solution}

Because we only possess one loop results for the structure functions, we must solve (2.11) perturbatively by expanding the graviton field and the structure functions in loop orders,

$$
\begin{aligned}
h_{\mu \nu}(x) & =h_{\mu \nu}^{(0)}(x)+h_{\mu \nu}^{(1)}(x)+h_{\mu \nu}^{(2)}(x)+\ldots \\
F_{0,2}\left(x ; x^{\prime}\right) & =0+F_{0,2}^{(1)}\left(x ; x^{\prime}\right)+F_{0,2}^{(2)}\left(x ; x^{\prime}\right)+\ldots \\
G_{0,2}\left(x ; x^{\prime}\right) & =0+G_{0,2}^{(1)}\left(x ; x^{\prime}\right)+G_{0,2}^{(2)}\left(x ; x^{\prime}\right)+\ldots
\end{aligned}
$$

By substituting (2.12)-(2.14) into (2.11), we obtain equations for the tree order field $h_{\mu \nu}^{(0)}$ and the one-loop field $h_{\mu \nu}^{(1)}$,

$$
\begin{aligned}
E^{\mu \nu(0)}(x)= & \frac{\kappa}{2} \mathcal{T}_{\operatorname{lin}}^{\mu \nu}(x) \\
E^{\mu \nu(1)}(x)= & \mathcal{F}^{\mu \nu} \int d^{4} x^{\prime} i F_{0}^{(1)}\left(x ; x^{\prime}\right) \widetilde{R}_{\operatorname{lin} 0}\left(x^{\prime}\right)+\mathcal{G}^{\mu \nu} \int d^{4} x^{\prime} i G_{0}^{(1)}\left(x ; x^{\prime}\right) \overline{\widetilde{R}}_{\operatorname{lin} 0}\left(x^{\prime}\right) \\
& -2 \partial_{\alpha} \partial_{\beta} \int d^{4} x^{\prime}\left[i F_{2}^{(1)}\left(x ; x^{\prime}\right) \widetilde{C}_{\operatorname{lin} 0}^{\mu \alpha \beta}\left(x^{\prime}\right)+i G_{2}^{(1)}\left(x ; x^{\prime}\right) \widetilde{\widetilde{C}}_{\operatorname{lin} 0}^{\mu \alpha \nu \beta}\left(x^{\prime}\right)\right] \\
& +\left[\eta^{\mu \nu} \partial_{k} \partial_{\ell}-2 \delta_{(k}^{(\mu} \partial^{\nu)} \partial_{\ell)}+\delta_{k}^{(\mu} \delta_{\ell}^{\nu)} \partial^{2}\right] \int d^{4} x^{\prime} i G_{2}^{(1)}\left(x ; x^{\prime}\right) \widetilde{C}_{\operatorname{lin} 0}^{0 k 0 \ell}\left(x^{\prime}\right) \equiv \mathcal{S}^{\mu \nu}(x)
\end{aligned}
$$

Here $E^{\mu \nu(\ell)} \equiv \mathcal{D}^{\mu \nu \rho \sigma} h_{\rho \sigma}^{(\ell)}$. Note that in (2.15) we regard the matter source as 0th order, assuming the stress tensor includes no loop corrections from the 1PI 1-point function. The solution of the 0th order equation $h_{\mu \nu}^{(0)}$ enters the right hand side of the 1st order equation (2.16) to provide sources for the one-loop field $h_{\mu \nu}^{(1)}$.

\subsection{Schwinger-Keldysh formalism}

The perturbative effective field equation (2.16) seems to be ready for use, but if one were to interpret it in the spirit of the in-out formalism it would possess two disturbing features: 
- Acausality: the in-out effective field equation at $x^{\mu}$ receives influence from points $x^{\prime \mu}$ which lie in the future of $x^{\mu}$, and at spacelike separation from it.

- Imaginary parts: the in-out effective field develops an imaginary part if there is particle production.

Neither of these features prevents one from describing flat space scattering problems, but they would be problematic for cosmological settings in which we do not know what happens in the asymptotic future and the more natural question is how the fields evolve when released at finite time in some prepared state. That question is answered by the SchwingerKeldysh formalism [18-22]. This technique produces true expectation values, rather than in-out matrix elements, so the effective field equations at $x^{\mu}$ depend only on points $x^{\prime \mu}$ on or within its past light-cone, and the effective fields associated with Hermitian operators are real. Because excellent reviews on the Schwinger-Keldysh formalism exist [68-71], and the current authors have described it before in [55], we merely comment that the linearized Schwinger-Keldysh effective field equation is obtained by replacing the in-out self-energy with its retarded counterpart,

$$
\left[{ }^{\mu \nu} \Sigma^{\rho \sigma}\right]\left(x ; x^{\prime}\right) \rightarrow\left[{ }^{\mu \nu} \sum_{\text {Ret }}^{\rho \sigma}\right]\left(x ; x^{\prime}\right) \equiv\left[{ }^{\mu \nu} \sum_{++}^{\rho \sigma}\right]\left(x ; x^{\prime}\right)+\left[{ }^{\mu \nu} \Sigma_{+-}^{\rho \sigma}\right]\left(x ; x^{\prime}\right) .
$$

In this expression, we obtain $\left[{ }^{\mu \nu} \Sigma_{++}^{\rho \sigma}\right]$ and $\left[{ }^{\mu \nu} \Sigma_{+-}^{\rho \sigma}\right]$ from (2.8) by replacing the de Sitter length function $y\left(x ; x^{\prime}\right)$ by $y_{++}\left(x ; x^{\prime}\right)$ and $y_{+-}\left(x ; x^{\prime}\right)$, respectively, where

$$
\begin{aligned}
& y_{++}\left(x ; x^{\prime}\right) \equiv H^{2} a a^{\prime}\left[\left\|\vec{x}-\vec{x}^{\prime}\right\|^{2}-\left(\left|\eta-\eta^{\prime}\right|-i \varepsilon\right)^{2}\right]=y\left(x, x^{\prime}\right), \\
& y_{+-}\left(x ; x^{\prime}\right) \equiv H^{2} a a^{\prime}\left[\left\|\vec{x}-\vec{x}^{\prime}\right\|^{2}-\left(\eta-\eta^{\prime}+i \varepsilon\right)^{2}\right] .
\end{aligned}
$$

This converts the nonzero structure functions in (2.16) to the retarded ones of the Schwinger-Keldysh formalism [55],

$$
\begin{gathered}
F_{0}^{(1)}\left(x ; x^{\prime}\right)=\frac{i \kappa^{2}}{576 \pi^{3}}\left\{\frac{\partial^{4}-4 H^{2} a a^{\prime} \partial^{2}}{16}\left[\left[\ln \left(\frac{-y}{4 a a^{\prime}}\right)-1\right] \Theta\right]-\frac{1}{4} H^{2} a a^{\prime} \ln \left(a a^{\prime}\right) \partial^{2} \Theta\right. \\
\left.+H^{4} a^{2} a^{\prime 2}\left[3-\frac{1}{4-y}+\frac{3}{4}(2-y) \ln \left(\frac{-y}{4-y}\right)\right] \Theta\right\}, \\
F_{2}^{(1)}\left(x ; x^{\prime}\right)=\frac{i \kappa^{2}}{64 \pi^{3}}\left\{\frac{\partial^{4}+20 H^{2} a a^{\prime} \partial^{2}}{240}\left[\left[\ln \left(\frac{-y}{4 a a^{\prime}}\right)-1\right] \Theta\right]+\frac{H^{2} a a^{\prime} \ln \left(a a^{\prime}\right)}{12} \partial^{2} \Theta\right. \\
\left.+H^{4} a^{2} a^{\prime 2}\left[\frac{-\frac{1}{3}}{4-y}-\frac{1}{6} \ln \left(\frac{-y}{4-y}\right)\right] \Theta\right\} \\
G_{2}^{(1)}\left(x ; x^{\prime}\right)=\frac{i \kappa^{2}}{64 \pi^{3}}\left\{H^{4} a^{2} a^{\prime 2}\left[\frac{\frac{4}{3}}{4-y}+\frac{1}{3} \ln \left(\frac{-y}{4-y}\right)\right] \Theta\right\}
\end{gathered}
$$

(Note that $G_{0}^{(1)}\left(x ; x^{\prime}\right)$ is zero for the MMC scalar at one loop.) Here the symbol $\Theta$ stands for the Heaviside step function which ensures causality,

$$
\Theta \equiv \theta\left(\Delta \eta-\left\|\vec{x}-\vec{x}^{\prime}\right\|\right) \quad, \quad \Delta \eta \equiv \eta-\eta^{\prime},
$$


and now the $i \epsilon$-prescription can be dropped in $-y\left(x ; x^{\prime}\right)$ in eqs. $(2.20)-(2.22)$,

$$
-y\left(x ; x^{\prime}\right) \rightarrow-\left.y\left(x ; x^{\prime}\right)\right|_{\epsilon=0}=H^{2} a a^{\prime}\left[\Delta \eta^{2}-\left\|\vec{x}-\vec{x}^{\prime}\right\|^{2}\right] .
$$

Also note that the structure functions are pure imaginary, which makes the effective field equation (2.16) manifestly real. Therefore, the resulting Schwinger-Keldysh effective field equation is causal and real as promised.

\section{Quantum corrected gravitational potentials}

In this section we solve the effective field equations (2.15) and (2.16) which we repeat below,

$$
\begin{aligned}
& E^{\mu \nu(0)}(x)=\frac{\kappa}{2} \mathcal{T}_{\text {lin }}^{\mu \nu}(x) \\
& E^{\mu \nu(1)}(x)=\mathcal{S}^{\mu \nu}(x)
\end{aligned}
$$

with the retarded structure functions (2.20)-(2.22) for the graviton field. We are interested in quantum loop corrections to the gravitational response of a static point mass $M$. The 0th order equation (3.1) determines the classical response to a point particle. The 1st order equation (3.2) leads to the one-loop correction to the classical gravitational potentials.

\subsection{Classical solutions}

The linearized stress tensor density $\mathcal{T}_{\text {lin }}^{\mu \nu}(x)$ in (3.1) for a static point mass $M$ on the de Sitter background takes the form [72],

$$
\mathcal{T}_{\operatorname{lin}}^{\mu \nu}(x)=-a(\eta) \delta_{0}^{\mu} \delta_{0}^{\nu} M \delta^{3}(\vec{x}) .
$$

The symmetries of this system imply a solution of the form,

$$
h_{00}(x)=f_{1}(\eta, r), \quad h_{0 i}(x)=\partial_{i} f_{2}(\eta, r), \quad h_{i j}(x)=\delta_{i j} f_{3}(\eta, r)+\partial_{i} \partial_{j} f_{4}(\eta, r)
$$

where $r \equiv\|\vec{x}\|$. It is convenient to choose the longitudinal (Newtonian) gauge $f_{2}=0$ and $f_{4}=0 .^{2}$ In terms of these variables the $E^{\mu \nu}$ of expressions (3.1), (3.2) take the form,

$$
\begin{aligned}
E^{00}= & a^{2}\left\{-3 a^{2} H^{2} f_{1}+\left(\nabla^{2}-3 a H \partial_{0}\right) f_{3}\right\}, \\
E^{0 i}= & a^{2} \partial_{i}\left\{-a H f_{1}-\partial_{0} f_{3}\right\}, \\
E^{i j}= & a^{2} \partial_{i} \partial_{j}\left\{-\frac{1}{2} f_{1}+\frac{1}{2} f_{3}\right\} \\
& +a^{2} \delta_{i j}\left\{\left(\frac{1}{2} \nabla^{2}+a H \partial_{0}+3 a^{2} H^{2}\right) f_{1}+\left(\partial_{0}^{2}+2 a H \partial_{0}-\frac{1}{2} \nabla^{2}\right) f_{3}\right\} .
\end{aligned}
$$

\footnotetext{
${ }^{2}$ Instead of completely gauge fixing, one could have employed the gauge invariant formalism analogous to refs. $[24,25]$. The final results, expressed through the one-loop corrected Bardeen potentials, can be easily related to the results obtained here. For the reasons of simplicity we shall not proceed here along this technically more involved path.
} 
The classical solutions of the 0th order equation (3.1) are

$$
\begin{aligned}
& f_{1}(x) \rightarrow f_{1}^{(0)}(x)=\frac{2 G M}{a(\eta)\|\vec{x}\|} \equiv-2 \phi^{(0)}(x), \\
& f_{3}(x) \rightarrow f_{3}^{(0)}(x)=\frac{2 G M}{a(\eta)\|\vec{x}\|} \equiv-2 \psi^{(0)}(x),
\end{aligned}
$$

where $\phi^{(0)}(x)$ and $\psi^{(0)}(x)$ are the usual potentials in the longitudinal gauge. Note that these classical solutions (3.8) are just conformally rescaled potentials of a point mass in Minkowski space. At the classical level, both $\mathcal{T}_{\text {lin }}$ and $E_{0}^{\mu \nu}$ with $f_{1,3}^{(0)}$ given by (3.8), obey the (covariant) conservation identities, $\partial_{\mu} \mathcal{T}_{\text {lin }}^{\mu \nu}+a H \delta_{0}^{\nu} \eta_{\alpha \beta} \mathcal{T}_{\text {lin }}^{\alpha \beta}=0$ and $\partial_{\mu} E_{0}^{\mu \nu}+a H \delta_{0}^{\nu} \eta_{\alpha \beta} E_{0}^{\alpha \beta}=0$.

\subsection{Computing the one-loop source integrals}

The one-loop source terms on the right hand side of (3.2) in the $3+1$ decomposition are

$$
\begin{aligned}
\mathcal{S}^{00}= & \frac{\kappa^{2} M}{2} \mathcal{F}^{00} \int \frac{d \eta^{\prime}}{a\left(\eta^{\prime}\right)}\left[i F_{0}^{(1)}\left(x, x^{\prime}\right)\right]_{\vec{x}^{\prime}=0} \\
& -\frac{\kappa^{2} M}{3} \nabla^{2} \int \frac{d \eta^{\prime}}{a\left(\eta^{\prime}\right)}\left[i F_{2}^{(1)}\left(x, x^{\prime}\right)+\frac{1}{2} i G_{2}^{(1)}\left(x, x^{\prime}\right)\right]_{\vec{x}^{\prime}=0}, \\
\mathcal{S}^{0 i}= & \frac{\kappa^{2} M}{2} \mathcal{F}^{0 i} \int \frac{d \eta^{\prime}}{a\left(\eta^{\prime}\right)}\left[i F_{0}^{1}\left(x, x^{\prime}\right)\right]_{\vec{x}^{\prime}=0} \\
& +\frac{\kappa^{2} M}{3} \partial_{0} \partial_{i} \int \frac{d \eta^{\prime}}{a\left(\eta^{\prime}\right)}\left[i F_{2}^{(1)}\left(x, x^{\prime}\right)+\frac{1}{2} i G_{2}^{(1)}\left(x, x^{\prime}\right)\right]_{\vec{x}^{\prime}=0}, \\
\mathcal{S}^{i j}= & \frac{\kappa^{2} M}{2} \mathcal{F}^{i j} \int \frac{d \eta^{\prime}}{a\left(\eta^{\prime}\right)}\left[i F_{0}^{(1)}\left(x, x^{\prime}\right)\right]_{\vec{x}^{\prime}=0} \\
& -2 \partial_{0}^{2} \int d^{4} x^{\prime}\left[F_{2}^{(1)}\left(x ; x^{\prime}\right)+\frac{1}{2} G_{2}^{(1)}\left(x ; x^{\prime}\right)\right] \frac{G M}{a^{\prime}}\left[-\partial_{i}^{\prime} \partial_{j}^{\prime}+\frac{1}{3} \delta_{i j} \nabla^{\prime 2}\right] \frac{1}{\left\|\vec{x}^{\prime}\right\|} \\
& -\frac{\kappa^{2} M}{6}\left[\delta^{i j} \nabla^{2}-\partial^{i} \partial^{j}\right] \int \frac{d \eta^{\prime}}{a\left(\eta^{\prime}\right)}\left[F_{2}^{(1)}\left(x ; x^{\prime}\right)+\frac{1}{2} G_{2}^{(1)}\left(x ; x^{\prime}\right)\right]_{\vec{x}^{\prime}=0},
\end{aligned}
$$

where

$$
\begin{aligned}
\mathcal{F}^{00} & =a^{2}\left[\nabla^{2}-3 a H \partial_{0}+3 a^{2} H^{2}\right] a^{-2}=\nabla^{2}-3 a H \partial_{0}+9 a^{2} H^{2}, \\
\mathcal{F}^{0 i} & =a^{2} \partial_{i}\left[-\partial_{0}+a H\right] a^{-2}=\partial_{i}\left[-\partial_{0}+3 a H\right] \\
\mathcal{F}^{i j} & =a^{2}\left[-\left(\delta_{i j} \nabla^{2}-\partial_{i} \partial_{j}\right)+\delta_{i j}\left(\partial_{0}^{2}+a H \partial_{0}-3 a^{2} H^{2}\right)\right] a^{-2}, \\
& =-\left(\delta_{i j} \nabla^{2}-\partial_{i} \partial_{j}\right)+\delta_{i j}\left(\partial_{0}^{2}-3 a H \partial_{0}-3 a^{2} H^{2}\right) .
\end{aligned}
$$

One can check that the left hand side of the effective equation with arbitrary functions $f_{1,3}$ obey a conservation identity,

$$
\partial_{\mu} E^{\mu \nu}+a H \delta_{0}^{\nu} \eta_{\alpha \beta} E^{\alpha \beta}=0
$$

which is a consequence of the contracted linear Bianchi identity. Because of the special (transverse) character of $\mathcal{F}^{\mu \nu}$ in eqs. (3.13)-(3.15), an analogous conservation identity holds 
for the right hand side $\mathcal{S}^{\mu \nu}$ with an arbitrary choice of $F_{0}^{(1)}, F_{2}^{(1)}$ and $G_{2}^{(1)}$. These represent a nontrivial check of our equations. Moreover, these tell us that the four equations are not independent. One can solve any two equations; the other two follow from the conservation identities. (Had one proceeded with the gauge invariant formulation, one would need to cleverly combine the four equations into two gauge invariant equations, resulting in the two equations for gauge invariant scalar potentials.)

There is one ugly term on the right hand side of the second line of the $(i j)$ equation. All other terms contain only time integrations, but that term requires a three dimensional spatial integration. It is hence worth spending some effort and analyse all four equations, to see whether we can get rid of the spatial integration when evaluating the one-loop corrected $f_{1,3}$. These equations can be easily obtained from the $(00),(0 i)$ and $(i j)$ equations given in eqs. (3.5)-(3.7) and (3.10)-(3.15), ${ }^{3}$

$$
\begin{aligned}
-3 a^{2} H^{2} f_{1}^{(1)}+ & \left(\nabla^{2}-3 a H \partial_{0}\right) f_{3}^{(1)} \\
= & \frac{\kappa^{2} M}{2 a^{2}}\left[\nabla^{2}-3 a H \partial_{0}+9 a^{2} H^{2}\right] \int \frac{d \eta^{\prime}}{a\left(\eta^{\prime}\right)}\left[i F_{0}^{(1)}\left(x, x^{\prime}\right)\right]_{\vec{x}^{\prime}=0} \\
& -\frac{\kappa^{2} M}{3 a^{2}} \nabla^{2} \int \frac{d \eta^{\prime}}{a\left(\eta^{\prime}\right)}\left[i F_{2}^{(1)}\left(x, x^{\prime}\right)+\frac{1}{2} i G_{2}^{(1)}\left(x, x^{\prime}\right)\right]_{\vec{x}^{\prime}=0}, \\
-a H f_{1}^{(1)}-\partial_{0} f_{3}^{(1)} & \\
= & \frac{\kappa^{2} M}{2 a^{2}}\left[-\partial_{0}+3 a H\right] \int \frac{d \eta^{\prime}}{a\left(\eta^{\prime}\right)}\left[i F_{0}^{(1)}\left(x, x^{\prime}\right)\right]_{\vec{x}^{\prime}=0} \\
& +\frac{\kappa^{2} M}{3 a^{2}} \partial_{0} \int \frac{d \eta^{\prime}}{a\left(\eta^{\prime}\right)}\left[i F_{2}^{(1)}\left(x, x^{\prime}\right)+\frac{1}{2} i G_{2}^{(1)}\left(x, x^{\prime}\right)\right]_{\vec{x}^{\prime}=0} \\
-\frac{1}{2} f_{1}^{(1)}+\frac{1}{2} f_{3}^{(1)} & \frac{\kappa^{2} M}{2 a^{2}} \int \frac{d \eta^{\prime}}{a\left(\eta^{\prime}\right)}\left[i F_{0}^{(1)}\left(x, x^{\prime}\right)\right]_{\vec{x}^{\prime}=0} \\
& +\frac{2}{a^{2}} \partial_{0}^{2} \int d^{4} x^{\prime}\left[i F_{2}^{(1)}\left(x ; x^{\prime}\right)+\frac{1}{2} i G_{2}^{(1)}\left(x ; x^{\prime}\right)\right] \frac{G M}{a^{\prime}} \frac{1}{\left\|\vec{x}^{\prime}\right\|} \\
& +\frac{\kappa^{2} M}{6 a^{2}} \int \frac{d \eta^{\prime}}{a\left(\eta^{\prime}\right)}\left[i F_{2}^{(1)}\left(x ; x^{\prime}\right)+\frac{1}{2} i G_{2}^{(1)}\left(x ; x^{\prime}\right)\right]_{\vec{x}^{\prime}=0} \\
& +\frac{\kappa^{2} M}{6 a^{2}}\left(\partial_{0}^{2}-\nabla^{2}\right) \int \frac{d \eta^{\prime}}{a\left(\eta^{\prime}\right)}\left[i F_{2}^{(1)}\left(x ; x^{\prime}\right)+\frac{1}{2} i G_{2}^{(1)}\left(x ; x^{\prime}\right)\right]_{\vec{x}^{\prime}=0} \\
\left(\frac{1}{2} \nabla^{2}+a H \partial_{0}\right. & \left.+3 a^{2} H^{2}\right) f_{1}^{(1)}+\left(\partial_{0}^{2}+2 a H \partial_{0}-\frac{1}{2} \nabla^{2}\right) f_{3}^{(1)} \\
= & \frac{\kappa^{2} M}{2 a^{2}}\left[-\nabla^{2}+\partial_{0}^{2}-3 a H \partial_{0}-3 a^{2} H^{2}\right] \int \frac{d \eta^{\prime}}{a\left(\eta^{\prime}\right)}\left[i F_{0}^{(1)}\left(x, x^{\prime}\right)\right]_{\vec{x}^{\prime}=0}
\end{aligned}
$$

The third equation (3.19) tell us that in order to determine the gravitational slip (defined as the difference of the two potentials) one ought to perform both the integrals over time

\footnotetext{
${ }^{3}$ One can extract two equations from the $(i j)$ equation by acting with the projectors, $\delta_{i j}-\partial_{i} \partial_{j} / \nabla^{2}$ and $(1 / 3) \delta_{i j}-\partial_{i} \partial_{j} / \nabla^{2}$, which extract the terms $\propto \delta_{i j}$ and $\partial_{i} \partial_{j}$, respectively.
} 
$\eta^{\prime}$ and space $\vec{x}^{\prime}$. It is convenient to define the two source integrals, the one-loop spin zero $S_{0}^{(1)}$ and spin two, $S_{2}^{(1)}$, as follows,

$$
\begin{aligned}
S_{0}^{(1)}(x) & \equiv \int \frac{d \eta^{\prime}}{a\left(\eta^{\prime}\right)}\left[i F_{0}^{(1)}\left(x, x^{\prime}\right)\right]_{\vec{x}^{\prime}=0}, \\
S_{2}^{(1)}(x) & \equiv \int \frac{d \eta^{\prime}}{a\left(\eta^{\prime}\right)}\left[i F_{2}^{(1)}\left(x ; x^{\prime}\right)+\frac{1}{2} i G_{2}^{(1)}\left(x ; x^{\prime}\right)\right]_{\vec{x}^{\prime}=0},
\end{aligned}
$$

where

$$
\begin{gathered}
{\left[i F_{0}^{(1)}\left(x, x^{\prime}\right)\right]_{\vec{x}^{\prime}=0}=-\frac{\kappa^{2}}{64 \times 9 \pi^{3}}\left\{\frac{\partial^{4}-4 a a^{\prime} H^{2} \partial^{2}}{16}\left[\left(\ln \left(\frac{H^{2}\left(\Delta \eta^{2}-r^{2}\right)}{4}\right)-1\right) \theta(\Delta \eta-r)\right]\right.} \\
-\frac{1}{4} H^{2} a a^{\prime} \ln \left(a a^{\prime}\right) \partial^{2} \theta(\Delta \eta-r) \\
\left.+H^{4} a^{2} a^{\prime 2}\left[3-\frac{1}{4-y}+\frac{3}{4}(2-y) \ln \left(\frac{-y}{4-y}\right)\right] \theta(\Delta \eta-r)\right\} \\
{\left[i F_{2}^{(1)}\left(x ; x^{\prime}\right)+\frac{1}{2} i G_{2}^{(1)}\left(x ; x^{\prime}\right)\right]_{\vec{x}^{\prime}=0}} \\
=-\frac{\kappa^{2}}{64 \times 9 \pi^{3}}\left\{\frac{3 \partial^{4}+60 a a^{\prime} H^{2} \partial^{2}}{80}\left[\left(\ln \left(\frac{H^{2}\left(\Delta \eta^{2}-r^{2}\right)}{4}\right)-1\right) \theta(\Delta \eta-r)\right]\right. \\
\left.\quad+\frac{3}{4} H^{2} a a^{\prime} \ln \left(a a^{\prime}\right) \partial^{2} \theta(\Delta \eta-r)+H^{4} a^{2} a^{\prime 2}\left[\frac{3}{4-y}\right] \theta(\Delta \eta-r)\right\} .
\end{gathered}
$$

The equation for the gravitational slip (3.19) then becomes,

$$
\nabla^{2}\left(f_{3}^{(1)}-f_{1}^{(1)}\right)=\frac{\kappa^{2} M}{a^{2}} \nabla^{2} S_{0}^{(1)}(x)+\frac{\kappa^{2} M}{a^{2}}\left(-\partial_{0}^{2}+\frac{1}{3} \nabla^{2}\right) S_{2}^{(1)}(x) .
$$

The solutions for $f_{1}^{(1)}$ and $f_{3}^{(1)}$ are obtained by combining (3.17) and (3.18),

$$
\begin{aligned}
& f_{1}^{(1)}(x)=-\frac{\kappa^{2} M}{2 a^{2}} S_{0}^{(1)}(x)+\frac{\kappa^{2} M}{a^{2}}\left[-\frac{2}{3}+\nabla^{-2}\left(\partial_{0}^{2}-a H \partial_{0}\right)\right] S_{2}^{(1)}(x) \equiv-2 \phi^{(1)}(x), \\
& f_{3}^{(1)}(x)=\frac{\kappa^{2} M}{2 a^{2}} S_{0}^{(1)}(x)+\frac{\kappa^{2} M}{a^{2}}\left[-\frac{1}{3}-\nabla^{-2} a H \partial_{0}\right] S_{2}^{(1)}(x) \equiv-2 \psi^{(1)}(x),
\end{aligned}
$$

where

$$
\nabla^{-2} f(\eta, \vec{x})=-\frac{1}{4 \pi} \int d^{3} x^{\prime} \frac{f\left(\eta, \vec{x}^{\prime}\right)}{\left\|\vec{x}-\vec{x}^{\prime}\right\|} .
$$

By inserting (3.26) and (3.27) into (3.17)-(3.20) one sees that all of the equations (3.17)(3.20) are satisfied, representing a nontrivial check of our basic equations (3.26)-(3.27). We also see that the spatial integral of the spin two source is required for determination of the one-loop contributions to both gravitational potentials $\phi^{(1)}=-f_{1}^{(1)} / 2$ and $\psi^{(1)}=-f_{3}^{(1)} / 2$.

The actual calculation of the quantum (one-loop) corrected gravitational potentials $\phi^{(1)}$ and $\psi^{(1)}$ is rather technical and we relegate it to the appendix. Since the complete results are rather complex, in the last step of the calculation in the appendix we take 
the late time limiting form. The final result for $f_{1}^{(1)}$ and $f_{3}^{(1)}$ is given in eqs. (A.28)(A.29). From these, it is easy to extract the one-loop corrected potentials $\phi^{(1)}=-f_{1}^{(1)} / 2$, $\psi^{(1)}=-f_{3}^{(1)} / 2$. When $\kappa$ is replaced with $G$ (via the relation, $\kappa=\sqrt{16 \pi G}$ ) and the units $c, \hbar$ are appropriately re-inserted to elucidate the quantum gravitational nature of our calculation, we obtain from (A.28)-(A.29),

$$
\begin{aligned}
& \phi^{(1)}(x)=-\frac{G M}{a r}\left\{\frac{\hbar}{20 \pi c^{3}} \frac{G}{(a r)^{2}}\right.\left.+\frac{\hbar}{\pi c^{5}} G H^{2}\left[-\frac{1}{3} \ln (a)-\frac{3}{10} \ln \left(\frac{H r}{c}\right)+\mathcal{O}\left(\frac{1}{a^{3}}\right)\right]\right\} \\
& \psi^{(1)}(x)=-\frac{G M}{a r}\left\{-\frac{\hbar}{60 \pi c^{3}} \frac{G}{(a r)^{2}}\right. \\
&\left.\quad+\frac{\hbar}{\pi c^{5}} G H^{2}\left[-\frac{1}{3} \ln (a)-\frac{3}{10} \ln \left(\frac{H r}{c}\right)+\frac{2}{3} \frac{H a r}{c}+\mathcal{O}\left(\frac{1}{a^{3}}\right)\right]\right\} .
\end{aligned}
$$

It follows that MMC scalars in de Sitter background generate the conformal scalar contributions plus another positive contributions to the gravitational potentials. The first terms in eqs. (3.29)-(3.30) represent the one-loop contributions from a conformal scalar field, and in the limit when $H \rightarrow 0, a \rightarrow 1$ they reduce to the Minkowski space results of refs. [23-25], representing a nontrivial check of our principal results (3.29)-(3.30).

\section{Discussion}

We have included one loop effects from MMC scalars to derive quantum loop corrections to the potentials associated with a static point mass. Each of the full potentials, $\phi$ and $\psi$, can be presented as its classical value times a series of quantum corrections we have obtained in (3.29)-(3.30), which are at late times (when $a \gg 1$ ),

$$
\begin{aligned}
\phi_{\mathrm{dS}}(x)=-\frac{G M}{a r}\{1+ & \frac{\hbar}{20 \pi c^{3}} \frac{G}{(a r)^{2}} \\
& \left.+\frac{\hbar G H^{2}}{\pi c^{5}}\left[-\frac{1}{30} \ln (a)-\frac{3}{10} \ln \left(\frac{H a r}{c}\right)\right]+\mathcal{O}\left(G^{2}, \frac{1}{a^{3}}\right)\right\} \\
\psi_{\mathrm{dS}}(x)=-\frac{G M}{a r}\{1- & \frac{\hbar}{60 \pi c^{3}} \frac{G}{(a r)^{2}} \\
& \left.+\frac{\hbar G H^{2}}{\pi c^{5}}\left[-\frac{1}{30} \ln (a)-\frac{3}{10} \ln \left(\frac{H a r}{c}\right)+\frac{2}{3} \frac{H a r}{c}\right]+\mathcal{O}\left(G^{2}, \frac{1}{a^{3}}\right)\right\} .
\end{aligned}
$$

These can be compared with the corresponding flat space results which have been previously computed in [23-25],

$$
\begin{aligned}
& \phi_{\text {flat }}(x)=-\frac{G M}{r}\left\{1+\frac{\hbar}{20 \pi c^{3}} \frac{G}{r^{2}}+\mathcal{O}\left(G^{2}\right)\right\} \\
& \psi_{\text {flat }}(x)=-\frac{G M}{r}\left\{1-\frac{\hbar}{60 \pi c^{3}} \frac{G}{r^{2}}+\mathcal{O}\left(G^{2}\right)\right\} .
\end{aligned}
$$


From this comparison, one can see that the first quantum correction terms in (4.1)-(4.2) represent the de Sitter version of the flat space correction and the terms proportional to $G H^{2}$ are the intrinsic de Sitter corrections.

Note that every factor of the co-moving distance $r$ which appears in expressions (4.1)(4.2) is multiplied by a scale factor $a(\eta)$ so that their product gives the physical distance from source to observation point. The remaining factor of $\ln (a)$ multiplies a term of the same form as the classical potential. Because these secular terms contribute equally to both potentials, they can be reinterpreted as a time dependent renormalization of the mass term,

$$
M \rightarrow M\left[1-\frac{\hbar}{c^{5}} \frac{G H^{2}}{30 \pi} \ln (a)\right]
$$

or equivalently a time dependent renormalization of the Newton's constant,

$$
G \rightarrow G\left[1-\frac{\hbar}{c^{5}} \frac{G H^{2}}{30 \pi} \ln (a)\right] .
$$

Even though the secular terms are suppressed by the loop counting parameter, $\hbar G H^{2} / \mathrm{c}^{5}$, whose value is less than $10^{-10}$ for primordial inflation, they are growing in time, and can eventually become large. Indeed, when the number of e-foldings, $\ln (a)=H t$ becomes of the order $c^{5} /\left(\hbar G H^{2}\right)$, the correction becomes large, signifying a breakdown of perturbation theory in the sense that, when $\left[\hbar G H^{2} / c^{5}\right] \ln (a) \sim 1$, all orders contribute significantly. To understand what happens at very late times, one would have to sum these higher loop contributions, which is a major unsolved problem [44-46, 73, 74].

The secular screening effect we have just described is fascinating. It might represent the seed of an explanation for why the Newton constant seems so much smaller than any other length scale of fundamental theory. However, there is no avoiding the sense of strangeness. If we adopt the perspective of an observer at fixed co-moving position, whose physical distance to the source increases exponentially in co-moving time, then quantum scalar fluctuations are erasing the gravitational imprint of a point source faster than its classical redshift, in precisely the region where the source has almost no effect. From the perspective of an observer at fixed physical distance (in static coordinates) one wonders why anything is changing at all.

The sense of strangeness is even stronger when we compare with the recent result [67] for one loop corrections to the gravitational potentials from virtual photons. Unlike our case of MMC scalars, conformal invariance means that photons behave the same, in de Sitter conformal coordinates, as they do in flat space. Yet they also induce secular screening [67]. From this we can infer that the screening effect originates not so much from the way quantum fluctuations are affected by inflation but rather from the different way that gravity responds to sources on de Sitter space as opposed to flat space.

Finally, it is interesting to speculate that quantum corrections to gravity from the epoch of primordial inflation might modify late time gravity in observable ways, for example, as regards explaining the current phase of cosmic acceleration. These type of questions have been investigated in the context of Einstein's gravity endowed with a non-minimally coupled, light scalar [75-77], as well as in the context of some simple non-local extensions of gravity [78-81]. 


\section{Acknowledgments}

We are grateful for conversations on this subject with M. Fröb and E. Verdaguer. This work was partially supported by KASI, by the D-ITP consortium, a program of the NWO that is funded by the Dutch Ministry of Education, Culture and Science (OCW), by NSF grants PHY-1205591 and PHY-1506513, and by the Institute for Fundamental Theory at the University of Florida.

\section{A Evaluating the source integral}

In this appendix we give some details on how to perform the source integrals $S_{0}^{(1)}$ and $S_{2}^{(1)}$ given in eqs. (3.21)-(3.22) and (3.23)-(3.24), which through eqs. (3.26)-(3.27) allow us to calculate the one-loop corrected scalar gravitational potentials $\phi^{(1)}=-f_{1}^{(1)} / 2$ and $\psi^{(1)}=-f_{3}^{(1)} / 2$.

To begin with, it is convenient to break the source integrals, $S_{0}^{(1)}$ and $S_{2}^{(1)}$ in eqs. (3.21)(3.22) into the following simpler integrals,

$$
\begin{aligned}
& \mathcal{I}_{1 a} \equiv \frac{1}{16} \partial^{4} \int_{\eta_{0}=-1 / H}^{\eta-r} \frac{d \eta^{\prime}}{a^{\prime}}\left[\ln \left(\frac{-y}{4 a a^{\prime}}\right)-1\right]=\frac{1}{4 a r^{3}} \\
& \mathcal{I}_{1 b} \equiv-\frac{H^{2} a}{4} \partial^{2} \int_{\eta_{0}=-1 / H}^{\eta-r} d \eta^{\prime}\left[\ln \left(\frac{-y}{4 a a^{\prime}}\right)-1\right] \\
&=\frac{H^{2} a}{r}\left\{\ln (H r)+\frac{1}{2} \ln \left(\frac{1-H r-\frac{1}{a}}{1+H r-\frac{1}{a}}\right)\right\} \\
& \mathcal{I}_{2} \equiv-\frac{H^{2} a}{4}\left[\ln (a) \partial^{2} \int_{\eta_{0}=-1 / H}^{\eta-r} d \eta^{\prime}+\partial^{2} \int_{\eta_{0}=-1 / H}^{\eta-r} d \eta^{\prime} \ln \left(a^{\prime}\right)\right]=\frac{H^{2} a}{2 r} \ln \left(\frac{a}{H r+\frac{1}{a}}\right) \\
& \mathcal{I}_{3 a} \equiv H^{4} a^{2} \int_{\eta_{0}=-1 / H}^{\eta-r} d \eta^{\prime} a^{\prime}\left[-\frac{1}{4-y}\right]=-\frac{H^{2} a}{2 r}\left\{\ln (1+a H r)+\ln \left(\frac{1-H r+\frac{1}{a}}{1+H r+\frac{1}{a}}\right)\right\} \\
& \mathcal{I}_{3 b} \equiv 3 H^{4} a^{2} \int_{\eta_{0}=-1 / H}^{\eta-r} d \eta^{\prime} a^{\prime}\left[1+\frac{1}{4}(2-y) \ln \left(\frac{-y}{4-y}\right)\right]=\frac{3}{4} H^{3} a^{3}\left\{4 H r \ln \left(1+\frac{1}{H a r}\right)\right. \\
&\left.\quad-\left[(1+H r)^{2}-\frac{1}{a^{2}}\right] \ln \left(\frac{1+H r+\frac{1}{a}}{1+H r-\frac{1}{a}}\right)-\left[(1-H r)^{2}-\frac{1}{a^{2}}\right] \ln \left(\frac{1-H r+\frac{1}{a}}{1-H r-\frac{1}{a}}\right)\right\} .
\end{aligned}
$$

When written in terms of these integrals, the spin zero and spin two sources (3.21)-(3.22) and (3.23)-(3.24) are simply,

$$
\begin{aligned}
& S_{0}^{(1)}(t, r)=-\frac{\kappa^{2}}{64 \times 3 \pi^{3}}\left[\frac{1}{3} \mathcal{I}_{1 a}+\frac{1}{3}\left(\mathcal{I}_{1 b}+\mathcal{I}_{2}+\mathcal{I}_{3 a}\right)+\frac{1}{3} \mathcal{I}_{3 b}\right] \\
& S_{2}^{(1)}(t, r)=-\frac{\kappa^{2}}{64 \times 3 \pi^{3}}\left[\frac{1}{5} \mathcal{I}_{1 a}-\left(\mathcal{I}_{1 b}+\mathcal{I}_{2}+\mathcal{I}_{3 a}\right)\right]
\end{aligned}
$$

where

$$
\mathcal{I}_{1 b}+\mathcal{I}_{2}+\mathcal{I}_{3 a}=-\frac{H^{2} a}{r}\left\{\ln \left[1+\frac{1}{H a r}\right]+\frac{1}{2} \ln \left[\frac{\left(1+H r-\frac{1}{a}\right)\left(1-H r+\frac{1}{a}\right)}{\left(1+H r+\frac{1}{a}\right)\left(1-H r-\frac{1}{a}\right)}\right]\right\} .
$$


Now, it is convenient to re-express the scalar gravitational fields $f_{1}^{(1)}$ and $f_{3}^{(1)}$ in eqs. (3.26)-(3.27) as,

$$
\begin{aligned}
& f_{1}^{(1)}(x)=\kappa^{2} M\left[-\frac{1}{2 a^{2}} S_{0}^{(1)}(x)-\frac{2}{3 a^{2}} S_{2}^{(1)}(x)+\partial_{t}^{2} \nabla^{-2} S_{2}^{(1)}(x)\right] \\
& f_{3}^{(1)}(x)=\kappa^{2} M\left[\frac{1}{2 a^{2}} S_{0}^{(1)}(x)-\frac{1}{3 a^{2}} S_{2}^{(1)}(x)-H \partial_{t} \nabla^{-2} S_{2}^{(1)}(x)\right],
\end{aligned}
$$

where

$$
\begin{aligned}
& S_{0}^{(1)}=-\frac{\kappa^{2}}{64 \times 3 \pi^{3}}\left\{\frac{1}{12} \frac{a^{2}}{(a r)^{3}}+a^{2} H^{3}\left[-\frac{1}{3 H a r} \ln \left(1+\frac{1}{H a r}\right)+\text { Har } \ln \left(1+\frac{1}{H a r}\right)\right.\right. \\
& +\frac{1}{6 H a r} \ln \left(\frac{\left(1+H r+\frac{1}{a}\right)\left(1-H r-\frac{1}{a}\right)}{\left(1+H r-\frac{1}{a}\right)\left(1-H r+\frac{1}{a}\right)}\right)-\frac{a}{4}\left((1+H r)^{2}-\frac{1}{a^{2}}\right) \ln \left(\frac{1+H r+\frac{1}{a}}{1+H r-\frac{1}{a}}\right) \\
& \left.\left.-\frac{a}{4}\left((1-H r)^{2}-\frac{1}{a^{2}}\right) \ln \left(\frac{1-H r+\frac{1}{a}}{1-H r-\frac{1}{a}}\right)\right]\right\} \\
& S_{2}^{(1)}=-\frac{\kappa^{2}}{64 \times 3 \pi^{3}}\left\{\frac{1}{20} \frac{a^{2}}{(a r)^{3}}\right. \\
& \left.+a^{2} H^{3}\left[\frac{1}{H a r} \ln \left(1+\frac{1}{H a r}\right)+\frac{1}{2 H a r} \ln \left(\frac{\left(1+H r-\frac{1}{a}\right)\left(1-H r+\frac{1}{a}\right)}{\left(1+H r+\frac{1}{a}\right)\left(1-H r-\frac{1}{a}\right)}\right)\right]\right\} .
\end{aligned}
$$

To fully reconstruct the gravitational potentials (A.9)-(A.10) we also need to evaluate $\nabla^{-2} S_{2}^{(1)}$, which can be broken into three parts,

$$
\begin{array}{r}
\nabla^{-2} S_{2}^{(1)}=-\frac{\kappa^{2} H^{3}}{64 \times 3 \pi^{3}}\left\{\frac{1}{20 a H^{3}} \nabla^{-2}\left(\frac{1}{r^{3}}\right)+\nabla^{-2}\left[\frac{a}{H r} \ln \left(1+\frac{1}{H a r}\right)\right]\right. \\
\left.+\nabla^{-2}\left[\frac{a}{2 H r} \ln \left(\frac{\left(1+H r-\frac{1}{a}\right)\left(1-H r+\frac{1}{a}\right)}{\left(1+H r+\frac{1}{a}\right)\left(1-H r-\frac{1}{a}\right)}\right)\right]\right\} .
\end{array}
$$

There are three pieces on which the inverse Laplace operator act. One can check that the first evaluates to,

$$
\mathcal{J}_{1} \equiv \frac{1}{20 a H^{3}} \nabla^{-2}\left(\frac{1}{r^{3}}\right)=-\frac{1}{20 H^{3}} \frac{\ln (H r)}{a r},
$$

where, for convenience, we fixed the integration constant to be $-\ln (H) / r{ }^{4}$ Strictly speaking, when $\nabla^{2}$ acts on $-\ln (H) / r$ it generates a delta function, $\propto \delta^{3}(\vec{x})$, but that term can be subsumed in a (finite) renormalization of the Newton constant. To evaluate the other two terms in (A.12) the following integral representation can be used,

$$
\nabla^{-2} g(r)=\int_{0}^{r} d r^{\prime} r^{\prime}\left(1-\frac{r^{\prime}}{r}\right) g\left(r^{\prime}\right)
$$

\footnotetext{
${ }^{4}$ Other choices of integration constants are possible. The plausible one is, $-\ln (H a) / r$, since in this case the final answer depends on the physical distance ar only. However, an inspection of the constraint equation (3.20) shows that this choice is illegitimate. Indeed, since $\mathcal{J}_{1}$ contributes equally to both $f_{1}$ and $f_{3}$, but it does not contribute to the right hand side, $\mathcal{J}_{1}$ must satisfy, $\left(\partial_{0}^{2}+3 a H \partial_{0}+3 a^{2} H^{2}\right) \mathcal{J}_{1}=$ $a^{2}\left(\partial_{t}^{2}+4 H \partial_{t}+3 H^{2}\right) \mathcal{J}_{1}=0$, from which we conclude that the choice of the integration constant in (A.14) is the correct one.
} 
where $\left[r^{2} g(r)\right]_{r \rightarrow 0}$ must vanish. Applying this to the other two terms in (A.12) yields,

$$
\begin{aligned}
\mathcal{J}_{2} \equiv & \nabla^{-2}\left[\frac{a}{H r} \ln \left(1+\frac{1}{H a r}\right)\right] \\
= & \frac{a}{2 H^{3} r}\left(H r+\frac{1}{a}\right)^{2}\left[\ln \left(H r+\frac{1}{a}\right)-\frac{3}{2}\right]-\frac{a r}{2 H}\left[\ln (H r)-\frac{3}{2}\right] \\
& +\frac{1}{H^{2}}[\ln (a)+1]+\frac{1}{2 r a H^{3}}\left[\ln (a)+\frac{3}{2}\right] \\
\mathcal{J}_{3} \equiv & \nabla^{-2}\left[\frac{a}{2 H r} \ln \left(\frac{\left(1+H r-\frac{1}{a}\right)\left(1-H r+\frac{1}{a}\right)}{\left(1+H r+\frac{1}{a}\right)\left(1-H r-\frac{1}{a}\right)}\right)\right] \\
= & \frac{a}{4 H^{3} r}\left\{\left(1+H r-\frac{1}{a}\right)^{2}\left[\ln \left(1+H r-\frac{1}{a}\right)-\frac{3}{2}\right]+\left(1-H r+\frac{1}{a}\right)^{2}\left[\ln \left(1-H r+\frac{1}{a}\right)-\frac{3}{2}\right]\right. \\
& \left.\quad-\left(1+H r+\frac{1}{a}\right)^{2}\left[\ln \left(1+H r+\frac{1}{a}\right)-\frac{3}{2}\right]-\left(1-H r-\frac{1}{a}\right)^{2}\left[\ln \left(1-H r-\frac{1}{a}\right)-\frac{3}{2}\right]\right\} \\
& +\frac{a}{H^{2}}\left(1+\frac{1}{a}\right)\left[\ln \left(1+\frac{1}{a}\right)-1\right]-\frac{a}{H^{2}}\left(1-\frac{1}{a}\right)\left[\ln \left(1-\frac{1}{a}\right)-1\right] .
\end{aligned}
$$

In terms of these integrals, $\nabla^{-2} S_{2}^{(1)}$ is simply,

$$
\nabla^{-2} S_{2}^{(1)}=-\frac{\kappa^{2} H^{3}}{64 \times 3 \pi^{3}}\left(\mathcal{J}_{1}+\mathcal{J}_{2}+\mathcal{J}_{3}\right)
$$

According to the expressions for the scalar potentials $f_{1}^{(1)}$ and $f_{3}^{(1)}$ in (A.9)-(A.10) we need the following combinations of the sources $S_{0}^{(1)}$ and $S_{2}^{(1)}$,

$$
\begin{aligned}
-\frac{S_{0}^{(1)}}{2 a^{2}}- & \frac{2 S_{2}^{(1)}}{3 a^{2}}=\frac{\kappa^{2}}{128 \times 3 \pi^{3}}\left\{\frac{3}{20} \frac{1}{(a r)^{3}}+H^{3}\left[\frac{1}{H a r} \ln \left(1+\frac{1}{H a r}\right)+H a r \ln \left(1+\frac{1}{H a r}\right)\right.\right. \\
& +\frac{1}{2 H a r} \ln \left(\frac{\left(1+H r-\frac{1}{a}\right)\left(1-H r+\frac{1}{a}\right)}{\left(1+H r+\frac{1}{a}\right)\left(1-H r-\frac{1}{a}\right)}\right)-\frac{a}{4}\left((1+H r)^{2}-\frac{1}{a^{2}}\right) \ln \left(\frac{1+H r+\frac{1}{a}}{1+H r-\frac{1}{a}}\right) \\
& \left.\left.-\frac{a}{4}\left((1-H r)^{2}-\frac{1}{a^{2}}\right) \ln \left(\frac{1-H r+\frac{1}{a}}{1-H r-\frac{1}{a}}\right)\right]\right\}, \\
\frac{S_{0}^{(1)}}{2 a^{2}}- & \frac{S_{2}^{(1)}}{3 a^{2}}=\frac{\kappa^{2}}{128 \times 3 \pi^{3}}\left\{-\frac{1}{20} \frac{1}{(a r)^{3}}+H^{3}\left[\frac{4}{3 H a r} \ln \left(1+\frac{1}{H a r}\right)-H a r \ln \left(1+\frac{1}{H a r}\right)\right.\right. \\
& +\frac{1}{2 H a r} \ln \left(\frac{\left(1+H r-\frac{1}{a}\right)\left(1-H r+\frac{1}{a}\right)}{\left(1+H r+\frac{1}{a}\right)\left(1-H r-\frac{1}{a}\right)}\right)+\frac{a}{4}\left((1+H r)^{2}-\frac{1}{a^{2}}\right) \ln \left(\frac{1+H r+\frac{1}{a}}{1+H r-\frac{1}{a}}\right) \\
& \left.\left.+\frac{a}{4}\left((1-H r)^{2}-\frac{1}{a^{2}}\right) \ln \left(\frac{1-H r+\frac{1}{a}}{1-H r-\frac{1}{a}}\right)\right]\right\} .
\end{aligned}
$$

\section{A.1 Late time limit of the gravitational potentials}

We now have all the ingredients to calculate $f_{1}^{(1)}$ and $f_{3}^{(1)}$ given in (A.9)-(A.10). The answer is rather long, and since we are primarily interested in the late time behavior, we now present the late time limit, $a \rightarrow \infty$, of various relevant contributions. First for the 
non-spatial integral terms in (A.9)-(A.10) we have

$$
\begin{gathered}
-\frac{S_{0}^{(1)}}{2 a^{2}}-\frac{2 S_{2}^{(1)}}{3 a^{2}} \stackrel{a \rightarrow \infty}{\longrightarrow} \frac{\kappa^{2} H^{3}}{64 \times 3 \pi^{3}}\left[\frac{3}{40(\text { Har })^{3}}-\frac{1}{4 H a r}+\frac{2}{3(H a r)^{2}}\right. \\
\left.-\frac{3}{8(H a r)^{3}}+\frac{4}{3\left(1-H^{2} r^{2}\right) a^{2}}+\mathcal{O}\left(\frac{1}{a^{4}}\right)\right] \\
\frac{S_{0}^{(1)}}{2 a^{2}}-\frac{S_{2}^{(1)}}{3 a^{2}} \stackrel{a \rightarrow \infty}{\longrightarrow} \frac{\kappa^{2} H^{3}}{64 \times 3 \pi^{3}}\left[\frac{-1}{40(H a r)^{3}}+\frac{1}{4 H a r}+\frac{1}{3(H a r)^{2}}-\frac{1}{8(H a r)^{3}}\right. \\
\left.+\frac{2}{3\left(1-H^{2} r^{2}\right) a^{2}}+\mathcal{O}\left(\frac{1}{a^{4}}\right)\right]
\end{gathered}
$$

For the spatial integral terms (A.16)-(A.17) which eventually enter (A.9)-(A.10), we obtain

$$
\begin{aligned}
& \mathcal{J}_{2} \stackrel{a \rightarrow \infty}{\longrightarrow} \frac{1}{H^{2}}\left\{\ln (\text { Har })+\frac{1}{2 H a r}\left[\ln (\text { Har })+\frac{3}{2}\right]+\frac{1}{6(\text { Har })^{2}}-\frac{1}{24(H a r)^{3}}+\mathcal{O}\left(\frac{1}{a^{4}}\right)\right\} \\
& \mathcal{J}_{3} \stackrel{a \rightarrow \infty}{\longrightarrow} \frac{1}{H^{2}}\left\{\frac{1+H r}{H r} \ln (1+H r)+\frac{1-H r}{H r} \ln (1-H r)+2+\frac{1}{3\left(1-H^{2} r^{2}\right) a^{2}}+\mathcal{O}\left(\frac{1}{a^{4}}\right)\right\} .
\end{aligned}
$$

Then the action of the inverse Laplacian operator on the spin two source becomes in the large $a$ limit,

$$
\begin{aligned}
\lim _{a \rightarrow \infty} \nabla^{-2} S_{2}^{(1)}=-\lim _{a \rightarrow \infty} \frac{\kappa^{2} H^{3}}{64 \times 3 \pi^{3}}\left(\mathcal{J}_{1}+\mathcal{J}_{2}+\mathcal{J}_{3}\right) \\
=\frac{\kappa^{2} H}{64 \times 3 \pi^{3}}\left\{\frac{\ln (H r)}{20 H a r}-\ln (\text { Har })-\frac{1}{2 H a r}\left[\ln (\text { Har })+\frac{3}{2}\right]-\frac{1}{6(H a r)^{2}}\right. \\
+\frac{1}{24(H a r)^{3}}-\frac{1+H r}{H r} \ln (1+H r)-\frac{1-H r}{H r} \ln (1-H r) \\
\left.-2-\frac{1}{3\left(1-H^{2} r^{2}\right) a^{2}}+\mathcal{O}\left(\frac{1}{a^{4}}\right)\right\}
\end{aligned}
$$

What enters $f_{1}^{(1)}$ and $f_{3}^{(1)}$ are the second and first derivative of this expression, respectively, i.e.

$$
\begin{gathered}
\partial_{t}^{2} \nabla^{-2} S_{2}^{(1)} \stackrel{a \rightarrow \infty}{\longrightarrow} \frac{\kappa^{2} H^{3}}{64 \times 3 \pi^{3}}\left\{\frac{\ln (\text { Hr })}{20 H a r}-\frac{1}{2 H a r}\left[\ln (\text { Har })-\frac{1}{2}\right]-\frac{2}{3(\text { Har })^{2}}+\frac{3}{8(\text { Har })^{3}}\right. \\
\left.-\frac{4}{3\left(1-H^{2} r^{2}\right) a^{2}}+\mathcal{O}\left(\frac{1}{a^{4}}\right)\right\}, \\
-H \partial_{t} \nabla^{-2} S_{2}^{(1)} \stackrel{a \rightarrow \infty}{\longrightarrow} \frac{\kappa^{2} H^{3}}{64 \times 3 \pi^{3}}\left\{\frac{\ln (H r)}{20 H a r}+1-\frac{1}{2 H a r}\left[\ln (\text { Har })+\frac{1}{2}\right]-\frac{1}{3(\text { Har })^{2}}+\frac{1}{8(\text { Har })^{3}}\right. \\
\left.-\frac{2}{3\left(1-H^{2} r^{2}\right) a^{2}}+\mathcal{O}\left(\frac{1}{a^{4}}\right)\right\} .
\end{gathered}
$$

Interestingly, all the negative powers of $a$ without the logarithm factors in (A.26)-(A.27) cancel the corresponding terms in (A.21)-(A.22). What finally remains in the scalar per- 
turbations $f_{1}^{(1)}$ and $f_{3}^{(1)}$ at late times are,

$$
\begin{aligned}
& f_{1}^{(1)}(x)=\kappa^{2} M \frac{\kappa^{2} H^{3}}{64 \times 3 \pi^{3}}\left[\frac{3}{40(\text { Har })^{3}}+\frac{\ln (H r)}{20 H a r}-\frac{\ln (\text { Har })}{2 H a r}+\mathcal{O}\left(\frac{1}{a^{4}}\right)\right] \\
& f_{3}^{(1)}(x)=\kappa^{2} M \frac{\kappa^{2} H^{3}}{64 \times 3 \pi^{3}}\left[-\frac{1}{40(\text { Har })^{3}}+\frac{\ln (H r)}{20 H a r}+1-\frac{\ln (\text { Har })}{2 H a r}+\mathcal{O}\left(\frac{1}{a^{4}}\right)\right] .
\end{aligned}
$$

These are our main results, which are used in the main text (3.29)-(3.30) to obtain the late time one-loop corrected gravitational potentials.

Open Access. This article is distributed under the terms of the Creative Commons Attribution License (CC-BY 4.0), which permits any use, distribution and reproduction in any medium, provided the original author(s) and source are credited.

\section{References}

[1] A.F. Radkowski, Some aspects of the source description of gravitation, Ann. Phys. 56 (1970) 319.

[2] D.M. Capper, M.J. Duff and L. Halpern, Photon corrections to the graviton propagator, Phys. Rev. D 10 (1974) 461 [INSPIRE].

[3] D.M. Capper and M.J. Duff, The one-loop neutrino contribution to the graviton propagator, Nucl. Phys. B 82 (1974) 147.

[4] D.M. Capper, On quantum corrections to the graviton propagator, Nuovo Cim. A 25 (1975) 29.

[5] M.J. Duff, Quantum corrections to the Schwarzschild solution, Phys. Rev. D 9 (1974) 1837 [INSPIRE].

[6] H.W. Hamber and S. Liu, On the quantum corrections to the Newtonian potential, Phys. Lett. B 357 (1995) 51 [hep-th/9505182] [INSPIRE].

[7] M.J. Duff and J.T. Liu, Complementarity of the Maldacena and Randall-Sundrum pictures, Class. Quant. Grav. 18 (2001) 3207 [hep-th/0003237] [INSPIRE].

[8] J.F. Donoghue, Leading quantum correction to the Newtonian potential, Phys. Rev. Lett. 72 (1994) 2996 [gr-qc/9310024] [INSPIRE].

[9] J.F. Donoghue, General relativity as an effective field theory: the leading quantum corrections, Phys. Rev. D 50 (1994) 3874 [gr-qc/9405057] [INSPIRE].

[10] I.J. Muzinich and S. Vokos, Long range forces in quantum gravity, Phys. Rev. D 52 (1995) 3472 [hep-th/9501083] [INSPIRE].

[11] A.A. Akhundov, S. Bellucci and A. Shiekh, Gravitational interaction to one loop in effective quantum gravity, Phys. Lett. B 395 (1997) 16 [gr-qc/9611018] [INSPIRE].

[12] I.B. Khriplovich and G.G. Kirilin, Quantum power correction to the Newton law, J. Exp. Theor. Phys. 95 (2002) 981 [gr-qc/0207118] [INSPIRE].

[13] I.B. Khriplovich and G.G. Kirilin, Quantum long range interactions in general relativity, eConf C 0306234 (2003) 1361 [gr-qc/0402018] [INSPIRE]. 
[14] N.E.J. Bjerrum-Bohr, J.F. Donoghue and B.R. Holstein, Quantum gravitational corrections to the nonrelativistic scattering potential of two masses, Phys. Rev. D 67 (2003) 084033 [Erratum ibid. D 71 (2005) 069903] [hep-th/0211072] [INSPIRE].

[15] D.A.R. Dalvit and F.D. Mazzitelli, Running coupling constants, Newtonian potential and nonlocalities in the effective action, Phys. Rev. D 50 (1994) 1001 [gr-qc/9402003] [InSPIRE].

[16] A. Satz, F.D. Mazzitelli and E. Alvarez, Vacuum polarization around stars: Nonlocal approximation, Phys. Rev. D 71 (2005) 064001 [gr-qc/0411046] [INSPIRE].

[17] D. Marolf, I.A. Morrison and M. Srednicki, Perturbative S-matrix for massive scalar fields in global de Sitter space, Class. Quant. Grav. 30 (2013) 155023 [arXiv:1209.6039] [InSPIRE].

[18] J.S. Schwinger, Brownian motion of a quantum oscillator, J. Math. Phys. 2 (1961) 407 [INSPIRE].

[19] K.T. Mahanthappa, Multiple production of photons in quantum electrodynamics, Phys. Rev. 126 (1962) 329 [INSPIRE].

[20] P.M. Bakshi and K.T. Mahanthappa, Expectation value formalism in quantum field theory. 1, J. Math. Phys. 4 (1963) 1 [inSPIRE].

[21] P.M. Bakshi and K.T. Mahanthappa, Expectation value formalism in quantum field theory. 2., J. Math. Phys. 4 (1963) 12 [inSPIRE].

[22] L.V. Keldysh, Diagram technique for nonequilibrium processes, Zh. Eksp. Teor. Fiz. 47 (1964) 1515 [INSPIRE].

[23] S. Park and R.P. Woodard, Solving the effective field equations for the Newtonian potential, Class. Quant. Grav. 27 (2010) 245008 [arXiv: 1007.2662] [INSPIRE].

[24] A. Marunovic and T. Prokopec, Time transients in the quantum corrected Newtonian potential induced by a massless nonminimally coupled scalar field, Phys. Rev. D 83 (2011) 104039 [arXiv: 1101.5059 ] [INSPIRE].

[25] A. Marunovic and T. Prokopec, Antiscreening in perturbative quantum gravity and resolving the Newtonian singularity, Phys. Rev. D 87 (2013) 104027 [arXiv:1209.4779] [inSPIRE].

[26] A.A. Starobinsky, Spectrum of relict gravitational radiation and the early state of the universe, JETP Lett. 30 (1979) 682 [Pisma Zh. Eksp. Teor. Fiz. 30 (1979) 719] [InSPIRE].

[27] V.F. Mukhanov and G.V. Chibisov, Quantum fluctuation and nonsingular universe (in Russian), JETP Lett. 33 (1981) 532 [Pisma Zh. Eksp. Teor. Fiz. 33 (1981) 549] [InSPIRE].

[28] S.W. Hawking, The development of irregularities in a single bubble inflationary universe, Phys. Lett. B 115 (1982) 295 [INSPIRE].

[29] A.H. Guth and S.Y. Pi, Fluctuations in the new inflationary universe, Phys. Rev. Lett. 49 (1982) 1110 [INSPIRE].

[30] A.A. Starobinsky, Dynamics of phase transition in the new inflationary universe scenario and generation of perturbations, Phys. Lett. B 117 (1982) 175 [INSPIRE].

[31] J.M. Bardeen, P.J. Steinhardt and M.S. Turner, Spontaneous creation of almost scale - Free density perturbations in an inflationary universe, Phys. Rev. D 28 (1983) 679 [INSPIRE].

[32] V.F. Mukhanov, Gravitational instability of the universe filled with a scalar field, JETP Lett. 41 (1985) 493 [Pisma Zh. Eksp. Teor. Fiz. 41 (1985) 402] [inSPIRE]. 
[33] V.F. Mukhanov, H.A. Feldman and R.H. Brandenberger, Theory of cosmological perturbations. Part 1. Classical perturbations. Part 2. Quantum theory of perturbations. Part 3. Extensions, Phys. Rept. 215 (1992) 203 [INSPIRE].

[34] T. Brunier, V.K. Onemli and R.P. Woodard, Two loop scalar self-mass during inflation, Class. Quant. Grav. 22 (2005) 59 [gr-qc/0408080] [InSPIRE].

[35] E.O. Kahya and V.K. Onemli, Quantum stability of a $w<-1$ phase of cosmic acceleration, Phys. Rev. D 76 (2007) 043512 [gr-qc/0612026] [INSPIRE].

[36] T. Prokopec, O. Tornkvist and R.P. Woodard, Photon mass from inflation, Phys. Rev. Lett. 89 (2002) 101301 [astro-ph/0205331] [INSPIRE].

[37] T. Prokopec, O. Tornkvist and R.P. Woodard, One loop vacuum polarization in a locally de Sitter background, Annals Phys. 303 (2003) 251 [gr-qc/0205130] [INSPIRE].

[38] T. Prokopec and R.P. Woodard, Vacuum polarization and photon mass in inflation, Am. J. Phys. 72 (2004) 60 [astro-ph/0303358] [INSPIRE].

[39] T. Prokopec and R.P. Woodard, Dynamics of superhorizon photons during inflation with vacuum polarization, Annals Phys. 312 (2004) 1 [gr-qc/0310056] [INSPIRE].

[40] T. Prokopec and E. Puchwein, Photon mass generation during inflation: de Sitter invariant case, JCAP 04 (2004) 007 [astro-ph/0312274] [INSPIRE].

[41] T. Prokopec and R.P. Woodard, Production of massless fermions during inflation, JHEP 10 (2003) 059 [astro-ph/0309593] [INSPIRE].

[42] B. Garbrecht and T. Prokopec, Fermion mass generation in de Sitter space, Phys. Rev. D 73 (2006) 064036 [gr-qc/0602011] [INSPIRE].

[43] L.D. Duffy and R.P. Woodard, Yukawa scalar self-mass on a conformally flat background, Phys. Rev. D 72 (2005) 024023 [hep-ph/0505156] [INSPIRE].

[44] S.-P. Miao and R.P. Woodard, The Fermion self-energy during inflation, Class. Quant. Grav. 23 (2006) 1721 [gr-qc/0511140] [InSPIRE].

[45] S.P. Miao and R.P. Woodard, Gravitons enhance fermions during inflation, Phys. Rev. D 74 (2006) 024021 [gr-qc/0603135] [INSPIRE].

[46] S.-P. Miao and R.P. Woodard, A simple operator check of the effective fermion mode function during inflation, Class. Quant. Grav. 25 (2008) 145009 [arXiv:0803.2377] [INSPIRE].

[47] E.O. Kahya and R.P. Woodard, Charged scalar self-mass during inflation, Phys. Rev. D 72 (2005) 104001 [gr-qc/0508015] [INSPIRE].

[48] E.O. Kahya and R.P. Woodard, One loop corrected mode functions for SQED during Inflation, Phys. Rev. D 74 (2006) 084012 [gr-qc/0608049] [INSPIRE].

[49] E.O. Kahya and R.P. Woodard, Quantum gravity corrections to the one loop scalar self-mass during inflation, Phys. Rev. D 76 (2007) 124005 [arXiv:0709.0536] [INSPIRE].

[50] E.O. Kahya and R.P. Woodard, Scalar field equations from quantum gravity during inflation, Phys. Rev. D 77 (2008) 084012 [arXiv:0710.5282] [INSPIRE].

[51] S. Park and R.P. Woodard, Scalar contribution to the graviton self-energy during inflation, Phys. Rev. D 83 (2011) 084049 [arXiv:1101.5804] [INSPIRE].

[52] S. Park and R.P. Woodard, Inflationary scalars don't affect gravitons at one loop, Phys. Rev. D 84 (2011) 124058 [arXiv:1109.4187] [INSPIRE]. 
[53] K.E. Leonard and R.P. Woodard, Graviton corrections to vacuum polarization during inflation, Class. Quant. Grav. 31 (2014) 015010 [arXiv: 1304.7265] [INSPIRE].

[54] P.J. Mora, N.C. Tsamis and R.P. Woodard, Hartree approximation to the one loop quantum gravitationalcorrection to the graviton mode function on de Sitter, JCAP 10 (2013) 018 [arXiv:1307.1422] [INSPIRE].

[55] K.E. Leonard, S. Park, T. Prokopec and R.P. Woodard, Representing the graviton self-energy on de Sitter background, Phys. Rev. D 90 (2014) 024032 [arXiv:1403.0896] [INSPIRE].

[56] S. Boran, E.O. Kahya and S. Park, Quantum gravity corrections to the conformally coupled scalar self-mass-squared on de Sitter background, Phys. Rev. D 90 (2014) 124054 [arXiv: 1409.7753] [INSPIRE].

[57] A. Campos and E. Verdaguer, Semiclassical equations for weakly inhomogeneous cosmologies, Phys. Rev. D 49 (1994) 1861 [gr-qc/9307027] [inSPIRE].

[58] A. Campos and E. Verdaguer, Stochastic semiclassical equations for weakly inhomogeneous cosmologies, Phys. Rev. D 53 (1996) 1927 [gr-qc/9511078] [INSPIRE].

[59] M.B. Fröb, A. Roura and E. Verdaguer, One-loop gravitational wave spectrum in de Sitter spacetime, JCAP 08 (2012) 009 [arXiv: 1205. 3097] [INSPIRE].

[60] M.B. Fröb, A. Roura and E. Verdaguer, Riemann correlator in de Sitter including loop corrections from conformal fields, JCAP 07 (2014) 048 [arXiv: 1403.3335] [INSPIRE].

[61] M.B. Fröb et al., Nonperturbative semiclassical stability of de Sitter spacetime for small metric deviations, Phys. Rev. D 87 (2013) 064019 [arXiv:1301.5261] [InSPIRE].

[62] D. Seery, One-loop corrections to a scalar field during inflation, JCAP 11 (2007) 025 [arXiv: 0707.3377] [INSPIRE].

[63] D. Seery, One-loop corrections to the curvature perturbation from inflation, JCAP 02 (2008) 006 [arXiv: 0707.3378] [INSPIRE].

[64] D. Seery, Infrared effects in inflationary correlation functions, Class. Quant. Grav. 27 (2010) 124005 [arXiv: 1005.1649] [INSPIRE].

[65] Y. Urakawa and K.-i. Maeda, One-loop corrections to scalar and tensor perturbations during inflation in stochastic gravity, Phys. Rev. D 78 (2008) 064004 [arXiv:0801.0126] [INSPIRE].

[66] T. Tanaka and Y. Urakawa, Loops in inflationary correlation functions, Class. Quant. Grav. 30 (2013) 233001 [arXiv: 1306.4461] [INSPIRE].

[67] C.L. Wang and R.P. Woodard, One-loop quantum electrodynamic correction to the gravitational potentials on de Sitter spacetime, Phys. Rev. D 92 (2015) 084008 [arXiv: 1508.01564] [INSPIRE].

[68] K.-c. Chou, Z.-b. Su, B.-l. Hao and L. Yu, Equilibrium and nonequilibrium formalisms made unified, Phys. Rept. 118 (1985) 1 [INSPIRE].

[69] R.D. Jordan, Effective field equations for expectation values, Phys. Rev. D 33 (1986) 444 [INSPIRE].

[70] E. Calzetta and B.L. Hu, Closed time path functional formalism in curved space-time: application to cosmological back reaction problems, Phys. Rev. D 35 (1987) 495 [INSPIRE].

[71] L.H. Ford and R.P. Woodard, Stress tensor correlators in the Schwinger-Keldysh formalism, Class. Quant. Grav. 22 (2005) 1637 [gr-qc/0411003] [INSPIRE]. 
[72] N.C. Tsamis and R.P. Woodard, The structure of perturbative quantum gravity on a de Sitter background, Commun. Math. Phys. 162 (1994) 217 [INSPIRE].

[73] H. Kitamoto and Y. Kitazawa, Non-linear $\sigma$-model in de Sitter space, Phys. Rev. D 83 (2011) 104043 [arXiv: 1012.5930] [INSPIRE].

[74] H. Kitamoto and Y. Kitazawa, Infra-red effects of non-linear $\sigma$-model in de Sitter space, Phys. Rev. D 85 (2012) 044062 [arXiv:1109.4892] [INSPIRE].

[75] D. Glavan, T. Prokopec and D.C. van der Woude, Late-time quantum backreaction from inflationary fluctuations of a nonminimally coupled massless scalar, Phys. Rev. D 91 (2015) 024014 [arXiv: 1408.4705 ] [INSPIRE].

[76] D. Glavan, T. Prokopec and T. Takahashi, Late-time quantum backreaction of a very light nonminimally coupled scalar [arXiv:1512.05329] [INSPIRE].

[77] D. Glavan, T. Prokopec and A. Starobinsky, Stochastic approach to late-time quantum backreaction, in preparation.

[78] S. Deser and R.P. Woodard, Nonlocal cosmology, Phys. Rev. Lett. 99 (2007) 111301 [arXiv: 0706.2151] [INSPIRE].

[79] S. Deser and R.P. Woodard, Observational viability and stability of nonlocal cosmology, JCAP 11 (2013) 036 [arXiv:1307.6639] [INSPIRE].

[80] S. Park and S. Dodelson, Structure formation in a nonlocally modified gravity model, Phys. Rev. D 87 (2013) 024003 [arXiv: 1209.0836] [INSPIRE].

[81] S. Dodelson and S. Park, Nonlocal gravity and structure in the universe, Phys. Rev. D 90 (2014) 043535 [arXiv:1310.4329] [INSPIRE]. 